\title{
Demander réparation(s). À Budapest, les mobilisations collectives à l'épreuve de leur visibilité
}

Demanding Compensation(s). Collective mobilizations testing their visibility in Budapest

Ludovic Lepeltier-Kutasi et Gergely Olt

\section{(2) OpenEdition \\ Journals}

\section{Édition électronique}

URL : http://journals.openedition.org/conflits/19165

DOI : 10.4000/conflits. 19165

ISSN : $1777-5345$

\section{Éditeur :}

CCLS - Centre d'études sur les conflits lilberté et sécurité, L'Harmattan

Édition imprimée

Date de publication : 19 mai 2016

Pagination : 81-98

ISBN : 978-2-343-09459-5

ISSN : $1157-996 X$

\section{Référence électronique}

Ludovic Lepeltier-Kutasi et Gergely Olt, « Demander réparation(s). À Budapest, les mobilisations collectives à l'épreuve de leur visibilité », Cultures \& Conflits [En ligne], 101 | printemps 2016, mis en ligne le 19 mai 2017, consulté le 30 mars 2021. URL : http://journals.openedition.org/conflits/19165 ; DOI : https://doi.org/10.4000/conflits.19165 


\section{Demander réparation(s). À Budapest, les mobilisations collectives à l'épreuve de leur visibilité}

\section{Ludovic LEPELTIER-KUTASI et Gergely OLT}

Ludovic Lepeltier-Kutasi est diplômé de l'EHESS, doctorant en géographie à l'Université François-Rabelais (UMR CITERES-EMAM), Tours.

Gergely Olt est doctorant en sociologie à l'Université Loránd Eötvös, membre du Centre de recherche en sciences sociales de l'Académie hongroise des sciences (MTA/TK), Budapest. Principales publications récentes : Csizmady A., Olt G., "Culture of Urban Rehabilitation: A Case Study about the Conflicts in the Jewish Quarter of Budapest ", in Papp R., Kuligowski W., Sterile and Isolated? An Anthropology Today in Hungary and Poland, Budapest, TIPI, 2015. Csanádi G., Csizmady A., Olt G., "Urban Renewal and Gentrification in Budapest City Center ", in Szirmai V. (dir.), Urban Sprawl in Europe: Similarities or Differences?, Budapest, AulaKiadó, 2011.

T es effets sociaux des politiques de renouvellement urbain sont depuis Lquelques années largement documentés et analysés, que ce soit sous l'angle des phénomènes de marginalisation spatiale ${ }^{1}$, des luttes de place - lorsque la gentrification est avérée - entre gentrifieurs et gentrifiés ${ }^{2}$, ou encore du rapport des habitants au « changement urbain ${ }^{3}$ ». Ces politiques urbaines peuvent varier fortement d'un contexte à l'autre et puiser dans une diversité de registres d'action, allant de mesures coercitives (reposant par exemple sur le déplacement autoritaire de populations captives) à des dispositifs d'ingénierie

1. Sur la question de la marginalisation spatiale, nous mentionnerons ici un " cas extrême », celui des politiques de déplacement dans le contexte budapestois : Jelinek Cs., «The phenomena of displacement and relocation during the process of gentrification in Budapest ", Studia UBB Sociologia, vol. 2, 2010, pp. 105-116.

2. Tissot S., De bons voisins. Enquête dans un quartier de la bourgeoisie progressiste, Paris, Raisons d'agir, 2011.

3. Giroud M., Résister en habitant? Renouvellement urbain et continuités populaires en centre ancien (Berriat Saint-Bruno à Grenoble et Alcântara à Lisbonne), Thèse de géographie, Université de Poitiers, 2007. 
sociale, se réclamant souvent de l'idéologie de l'empowerment ${ }^{4}$. À ce titre, ces derniers revendiquent une conception douce du renouvellement urbain, insistant sur la dimension utilitariste du développement social, notamment pour lutter contre des phénomènes très localisés de ségrégation avancée. Quelque part entre l'empowerment «social-libéral » et sa version "managériale » 5 , le programme de « réhabilitation sociale Magdolna » mené dans un quartier populaire de Budapest vise depuis 2005 à transformer en profondeur son image et son ambiance, tout en maintenant les habitants sur place. À partir d'une étude de cas, celui d'un groupe de locataires engagés dans un bras de fer avec les pouvoirs publics pour régler plusieurs litiges liés à la rénovation de leurs logements, l'objet de cet article est d'explorer la manière dont ces politiques produisent des formes spécifiques d'exclusion ${ }^{6}$.

Le programme Magdolna débute en 2005 à l'échelle d'un secteur très dégradé du huitième arrondissement de Budapest, compris dans un trapèze allant de Mátyás tér à Teleki tér, bordé au nord par Népszínház utca et au sud par Baross utca. Il s'agit, après le projet du quartier Corvin, de la deuxième étape de la reconquête des quartiers anciens péricentraux, élaborée par la municipalité d'arrondissement à la fin des années 1990. Alors que d'autres secteurs, considérés par les pouvoirs publics comme taudifiés, font l'objet de programmes très radicaux de rénovation urbaine au début de la décennie 1990, le programme Magdolna est au contraire conçu comme l'expérimentation en Hongrie de politiques urbaines «douces », porteuses de transformation sociale. Plus de dix ans après la fin du communisme, dans un contexte où les pouvoirs publics importent à tout va des modèles d'action publique élaborés le plus souvent en Europe occidentale 7 , le programme Magdolna apparaît comme le laboratoire budapestois de la soziale Stadt allemande, elle-même inspirée du new public management anglo-saxon ${ }^{8}$. Ces dispositifs répondent à la fois à des enjeux de lutte contre la ségrégation socio-spatiale - formulée dans notre contexte sous le terme de « lutte contre la ghettoïsation » - et d'optimisation gestionnaire du parc de logements municipaux. La régie publique d'aménagement Rév8 se voit alors confier la mise en ouvre du projet, allant du pilotage technique à la relation avec les habitants, tant en termes d'incitation à la participation que de gestion quotidienne des conflits liés à la réhabilitation.

4. Bacqué M.-H., «Empowerment et politiques urbaines aux États-Unis », Géographie, économie, société, vol. 8, n¹, 2006, pp. 107-124.

5. Bacqué M.H., Biewener C., L'empowerment, une pratique émancipatrice, Paris, La Découverte, 2013.

6. Nous entendons ici l'exclusion au sens donné par Norbert Elias et John L. Scotson, c'est-àdire fondée sur des dynamiques d'interaction incrustées dans des rapports de pouvoir. $C f$. Elias N., Logiques de l'exclusion, Paris, 2014, Fayard.

7. Saïsset P., La Hongrie post-communiste comme opportunité : de la diffusion à l'abandon des modèles d'intervention urbaine de la Caisse des dépôts et consignations. Le cas de l'arrondissement de Ferencváros à Budapest (1988-2014), Mémoire de master en anthropologie politique, EHESS, Paris, 2014.

8. Cuny C., Changement urbain et démocratie participative à Berlin. Ethnographie du grand 
Lorsque nous effectuons notre enquête en 2013-2014, les conflits liés à la réhabilitation portent surtout sur les malfaçons constatées après la fin des travaux, lesquelles produisent parfois pour les habitants de véritables entraves à l'accomplissement du quotidien. L'interpellation des managers de quartier, souvent des professionnels de l'urbain convertis de fait en médiateurs sociaux, échappe au début aux efforts de cadrage de l'expression habitante, pensée par Rév8 sous la forme de forums de riverains. Habitués à des formes d'interpellation directe - souvent discrète - des relais locaux du pouvoir, ces habitants se voient contraints, à mesure de la mise en échec répétée de ces méthodes traditionnelles d'interpellation, de rendre publique leur mobilisation. Le gain de visibilité de la controverse transforme alors les enjeux mêmes du conflit. D'une part, la mise en dispositif forcée déplace celui-ci dans un théâtre dont les pouvoirs publics maîtrisent la configuration. D'autre part, elle transforme les conditions du rapport de force dans la mesure où les autres habitants du quartier sont «invités au spectacle». Dans pareil contexte scénique, la « demande de réparation » endosse, en plus de la dimension matérielle, une forte dimension symbolique. De ce point de vue, les habitants mobilisés sont amenés à gérer un dilemme de taille : lutter pour la reconnaissance du caractère légitime de revendications ordinaires, sans tomber pour autant dans l'écueil d'une « reconnaissance déformée » visant un collectif partageant des traits communs malgré lui ${ }^{9}$. En d'autres termes, il s'agit pour ces riverains se revendiquant de la classe moyenne, de miser sur l'efficacité d'une stratégie collective sans pour autant donner le sentiment de faire front commun avec les strates les plus marginalisées des bénéficiaires de logements municipaux.

Les matériaux que nous mobiliserons ici sont en grande partie issus d'une enquête menée entre fin 2013 et début 2014 dans un immeuble réhabilité de logements municipaux dans lequel la question des malfaçons s'est posée de façon particulièrement vive. Cette unité résidentielle ne peut pas être considérée comme représentative de la façon dont le programme de réhabilitation Magdolna a eu un impact sur les habitants du quartier, ni même les seuls locataires d'appartements publics ${ }^{10}$. Elle nous a simplement servi de point de

ensemble de Marzahn, Paris, Éditions de la Maison des sciences de l'homme, 2014.

9. Axel Honneth, représentant contemporain de l'École de Francfort, insiste sur la distinction entre la reconnaissance visant l'émancipation et les déformations de la reconnaissance, dont la mobilisation de la notion à des fins instrumentales, par exemple dans l'idéologie managériale. Cf. Honneth A., La société du mépris. Vers une nouvelle théorie critique, Paris, La Découverte, 2008.

10. De ce point de vue, bien que l'entrée par immeuble ressemble beaucoup à des méthodologies éprouvées notamment en histoire urbaine, le choix de l'unité résidentielle enquêtée est loin des préoccupations de représentativité chères à Jean-Luc Pinol mais se rapproche davantage du paradigme indiciaire de Carlo Ginzburg, qui a connu des développements théoriques importants, notamment sous l'intitulé de la " pensée par cas ». L'enjeu de cette approche est de proposer une voie dans la montée en généralités à partir de configurations singulières. $C f$. Ginzburg C., "Traces », in Mythes, emblèmes, traces, Paris, Flammarion, pp. 139-180 et Passeron J.-C., Revel J., " Penser par cas. Raisonner à partir de singularités ", in Penser par cas, Paris, Éditions de l'EHESS, 2005, pp. 9-44. 
départ pour observer des situations concrètes d'engagement des acteurs dans un contexte de conflit. La démarche mise en œuvre, résolument ethnographique, a permis de recueillir les discours et d'observer les pratiques de riverains engagés ensemble dans le bras de fer avec la municipalité et Rév8 ${ }^{11}$. Nous avons ensuite recoupé ces données avec des matériaux issus de nos terrains de thèse respectifs, au cours desquels nous avons été amenés à rencontrer séparément et de façon additionnée une soixantaine d'habitants du quartier (parmi lesquels des leaders associatifs impliqués dans la controverse) et des acteurs institutionnels (représentant la municipalité d'arrondissement et Rév8). Même si nos objets de recherche sont différents, le caractère qualitatif de nos méthodologies ainsi que le choix d'une échelle d'observation micro, ont permis sans difficulté les passerelles disciplinaires ${ }^{12}$.

\section{Les logements municipaux dégradés au cœur des stratégies de reconquête urbaine}

Le quartier Magdolna est une construction politico-administrative récente, actant en 2012 le dépeçage de l'ancien secteur de KözépsöJózsefváros, dont le toponyme renvoyait trop dans les esprits à une réputation sulfureuse acquise tout au long du XXe siècle. Ancien faubourg populaire de Budapest construit lors de l'Âge d'or de la révolution industrielle, de l'arrivée du chemin de fer dans les gares de Keleti et Józsefváros, ce secteur situé en dehors du grand boulevard circulaire de Budapest devient petit à petit l'un des principaux marches-pieds des commerçants et artisans de province à ce qui est encore la seconde capitale de l'empire austro-hongrois. Ce qui correspond au quartier Magdolna est alors plutôt connu pour le grand marché aux puces de Teleki tér et les troquets et hauts lieux de la vie nocturne de Népszínház utca, où de nombreuses minorités ethniques - au premier rang desquelles une communauté juive séfarade et des Roms musiciens - se côtoient jusqu'à la fin des années 194013 .

Après la Seconde guerre mondiale, $90 \%$ des immeubles sont durement touchés par les combats entre Allemands et Soviétiques. Dans les années 1950, la démographie du quartier évolue sensiblement : alors qu'une bonne partie de

11. Chaque discussion a duré entre une et deux heures et était à chaque fois structurée par des questions ouvertes, portant sur la trajectoire sociale, la biographie résidentielle, le rapport au voisinage, les effets des politiques de réhabilitation sociale, les relations avec la sphère institutionnelle, les actions menées dans le cadre de la résolution des conflits éventuels. Ces entretiens ont été complétés par l'observation commune de réunions publiques portant sur des problématiques abordées par ces habitants, notamment celle de la hausse des charges locatives ou des malfaçons survenues après réhabilitation.

12. La thèse de Ludovic Lepeltier-Kutasi porte, en géographie, sur les effets sociaux des politiques de renouvellement urbain dans le quartier Magdolna; celle de Gergely Olt sur la gentrification festive du septième arrondissement de Budapest.

13. Le recueil de nouvelles des frères Giorgio et Nicola Pressburger regorge d'histoires et anecdotes relatant cette atmosphère. $C f$. Pressburger G., Pressburger N., Histoires du buitième district, Lagrasse, Verdier, 1989. 
la communauté juive est décimée pendant l'Holocauste, de nombreux habitants délaissent le secteur après-guerre, notamment les artisans et commerçants. Les abords de Teleki tér restent dans leur jus à l'instar de l'ensemble de l'arrondissement jusque dans les années 1990, en raison notamment de la priorité donnée sous le communisme à la construction de grands ensembles modernes en périphérie. Y vivent comme de nos jours, à la fois des professions manuelles peu qualifiées, mais aussi des professions intellectuelles mieux considérées, dans une mixité sociale très souvent oubliée. Le délabrement avancé du bâti, combiné à des logements souvent trop exigus, freine considérablement le processus de privatisation des biens immobiliers dans la décennie post-communiste ${ }^{14}$. Y subsiste alors un stock résiduel d'anciens logements d'État passés sous propriété de la municipalité d'arrondissement. Leur concentration dans le huitième arrondissement de Budapest a peu d'équivalents en Hongrie, mais pendant longtemps, les pouvoirs publics locaux ne disposent pas des fonds nécessaires à leur réhabilitation.

Alors que beaucoup de copropriétés, notamment celles des autres quartiers anciens de Budapest parviennent à financer leur réhabilitation, le huitième arrondissement semble, par contraste, se dégrader à vitesse accélérée. Par peur supposée ou avérée d'une "ghettoïsation ", semblant réactiver l'imaginaire de « ghetto rom » qui a longtemps collé à ce bout de ville ${ }^{15}$, les pouvoirs municipaux parviennent, à la fin des années 1990, à enclencher un vaste programme de renouvellement urbain, grâce notamment à un ambitieux partenariat public-privé ${ }^{16}$. C'est ainsi que le quartier de Szigony, à deux kilomètres au sud, est entièrement rasé pour faire place à un grand complexe de bureaux et de logements haut standing : le quartier Corvin. Au début des années 2000 , l'opportunité de financements européens fléchés vers les quartiers en difficulté sociale permet à la municipalité d'arrondissement de faire de l'un des périmètres d'action du plan de reconquête urbaine - le périmètre Magdolna -, le laboratoire hongrois de dispositifs de "réhabilitation sociale ", censés associer les habitants à la définition et la mise en œuvre du projet urbain. Celui-ci est inspiré d'initiatives éparses, au premier rang desquelles la

14. Hegedüs J., "Social housing in Hungary » in Whitehead C., Scanlon K. J. (dir.), Social housing in Europe, Londres, London School of Economics and Political Science, 2007, pp. $105-117$.

15. Tábori Z., Magdolna-negyed [Quartier Magdolna], Budapest, Osiris, 2009. Si ces représentations prennent leurs racines dans l'histoire, en raison d'une installation ancienne de « Roms musiciens » (muzsikúscigányok), elles sont généralement largement fantasmées, dans la mesure où la part de ceux qui se revendiquent de la minorité rom ne dépasse jamais $4 \%$ dans aucun des arrondissements de Budapest. Même s'il faut prendre les comptages ethniques avec toutes les précautions nécessaires, les données statistiques montrent des ordres de grandeur particulièrement nets, $c f$. Vukovich G. (dir.), 2011. évi népszámlalás. 3. Területi adatok. 3.1 Budapest [Recensement de 2011, 3. Données locales, 3.1. Budapest], Budapest, Központi Statisztikai Hivatal, 2013.

16. Keresztely K. et al., Les politiques de renouvellement urbain des villes d'Europe centrale illustrées par la rébabilitation des quartiers existants: la ville de Budapest en Hongrie, Agence nationale pour l'amélioration de l'habitat, 2008. 
soziale Stadt (« ville sociale ») allemande, dont l'idée est de coupler des mesures classiques de réhabilitation (rénovation des infrastructures, requalification des espaces communs, embellissement des façades, mesures de police de l'espace) à des dispositifs favorisant le lien social (développement des activités socio-culturelles et périscolaires, soutien au tissu associatif, organisation de réunions publiques durant toute la durée du programme).

Au moment où nous réalisons nos entretiens, le programme de réhabilitation sociale en est à la mise en œuvre de sa troisième phase ${ }^{17}$. L'immeuble dans lequel nous faisons notre enquête est alors réhabilité depuis trois ans, tel que programmé dans la deuxième phase. Auparavant, les logements étaient ce que l'on appelle des « chambre-cuisine » (szoba-konyba) sans confort (nemkomfortos), c'est-à-dire sans salle d'eau ni toilettes ${ }^{18}$. Si les appartements ne sont pas forcément dégradés, les espaces communs - coursives, cours, cages d'escalier - le sont. Après le chantier, chaque logement est agrandi de façon à accueillir des sanitaires, tandis que les réseaux d'eau, de gaz et d'électricité sont remis aux normes, les espaces communs réhabilités et la façade rafraîchie. Durant les travaux, les locataires sont, soit dédommagés et quittent le giron du parc locatif municipal, soit relogés ailleurs, de façon temporaire ou définitive. Lorsque les quelques anciens locataires reviennent habiter dans l'immeuble, ils ne retournent pas dans leur ancien appartement, mais dans un logement redimensionné et adapté cette fois à la taille de leur foyer. Ils retrouvent également un immeuble majoritairement vidé de ses habitants, où le contact avec les quelques nouveaux voisins est encore timide.

\section{Raisons et enjeux de la colère}

«J'en ai ras-le-bol de Rév8, j'en ai ras-le-bol de la municipalité, j'en ai marre de l'État hongrois, parce qu'ils nous ont tellement mis dans la misère, que ce soit au niveau de la santé, financièrement, moralement, de toutes les façons possibles... ${ }^{19}$ »

Lorsque nous rencontrons cette ancienne habitante, venue se réinstaller seule dans un nouveau logement de l'immeuble, la coupe est pleine. Depuis son retour, les mauvaises surprises s'accumulent, ne lui laissant que peu de répit. Tout d'abord, les indices sur lesquels sont estimés les loyers des logements municipaux - à savoir ici le niveau de confort, d'équipements, la dimension de l'appartement, l'état de l'immeuble - ont sensiblement progressé, éle-

17. Les deux premières phases ont eu lieu entre 2005 et 2011. La troisième a démarré en 2013.

18. La loi LXXVIII/1993 définit cinq niveaux de confort, alliant des critères de superficie minimale à des critères d'équipements (sanitaires, eau chaude, etc.).

19. Entretien avec une habitante réalisé en décembre 2013. Citation originale : « Nekem elegem van a Rév8-ból, nekem elegem van az önkormányzatból, elegem van a magyar államból, mert lenyomoritottak bennünket egészségileg, anyagilag, lelkileg, mindenhogy ». Tous les extraits d'entretien ont été traduits par Ludovic Lepeltier-Kutasi. 
vant mécaniquement le montant des frais locatifs 20. Ensuite, la facture annuelle de gaz qu'elle a reçue quelques mois avant notre entretien est nettement plus élevée que ce qu'elle avait l'habitude de payer avant les travaux de réhabilitation. Cette hausse est liée à l'installation d'un nouveau système de chauffage collectif mal calibré aux besoins des habitants. Ce mauvais dimensionnement a fait passer les logements d'une tarification domestique à une tarification dite commerciale, bien plus onéreuse, et surtout non éligible aux abattements sociaux dont certains locataires dans le besoin pouvaient bénéficier. Lorsque nous nous rencontrons fin décembre 2013, personne ne s'explique les raisons de cette augmentation brutale, tandis que le bailleur municipal, qui assure le relais entre le fournisseur d'énergie et les locataires, réclame expressément le versement de son dû.

«Les retraités qui vivent des aides sociales touchent 75000 forints [un peu moins de 250 euros, ndt] par mois, et pourtant, même à Buda il n'y a pas des montants [locatifs] aussi élevés qu'ici. Les charges mensuelles s'élèvent à 75000 forints lorsque l'on n'a pas le statut de locataire social. Celui-ci est réexaminé chaque année et, lorsque nous ne satisfaisons plus les critères, alors nous devons payer l'intégralité de ces charges. Chaque année, ils introduisent des règles tellement insidieuses, de manière à ce qu'on ne puisse plus être éligible. Et a priori nous sommes partis de cet appartement avec un loyer social. Auparavant, nous étions considérés comme dans le besoin, et désormais, nous ne sommes éligibles qu'au loyer conventionné 21 ».

Pour cette habitante, au-delà de la réévaluation du montant socle de son loyer, c'est également le statut locatif qui change, en raison d'une évolution du règlement municipal fixant les critères d'éligibilité au logement social. Elle passe d'une location sociale à une location conventionnée, c'est-à-dire avec un loyer indexé uniquement sur les caractéristiques intrinsèques du logement, sans abattement social. Dans le cas de cette retraitée employée dans une cantine pour sans-abris, les conséquences sont lourdes au niveau financier. Elle ne constitue pas un cas à part, comme nous l'ont montré les autres entretiens réalisés dans le même immeuble.

20. La loi LXXVIII/1993 fixe trois statuts locatifs pour les logements publics. Les deux premiers statuts - la location sociale et la location conventionnée (költségelven) sont indexées sur les caractéristiques intrinsèques des logements (dimension, équipements, niveau de confort, état de l'immeuble...), la différence étant que les locataires sociaux ne payent qu'un pourcentage de la valeur estimée. Le troisième statut est la location aux conditions du marché, permettant souvent aux municipalités de financer le reste du parc public.

21. Entretien avec une habitante réalisé en décembre 2013. Citation originale : "Havi 75 ezer forint a segély, a nyugdíbólélö embereknek, Budán nincsolyan összeg, mint ami itt van. A havirezsi 75 ezerforintra jön ki, ha nincsen szociális lakbér. Márpedig évente felülvizsgálják, és ha nem felelünk meg a kritériumoknak, akkor a teljesösszeget kell fizetni. Már pedig évente hoznak olyan kegyetlen szabályokat, hogy ne feleljen meg azember. És eleve szociális lakbérrel mentünk el ebböl a házból. Eleve rászorulóként, és költségelvünek adták vissza. ». 
Un autre élément qui explique les raisons de la colère réside dans la façon même dont les appartements ont été rénovés. Outre les frais locatifs auxquels ils doivent faire face, les habitants qui ont pu revenir dans l'immeuble doivent se réinstaller dans des espaces redimensionnés, dans lesquels leurs meubles et effets personnels ne trouvent plus forcément leur place. Par ailleurs, chacun d'eux insiste sur la découverte de « mauvaises surprises » qui posent de sérieux problèmes à l'accomplissement du quotidien. Celles-ci renvoient à des malfaçons techniques, renvoyant soit au choix de matériaux de mauvaise qualité, soit à l'absence de travaux de finition. À titre d'exemples, les nouvelles cloisons ne peuvent plus recevoir de rangements en hauteur, tandis que la mauvaise fermeture des portes et fenêtres pose des problèmes d'isolation. Par ailleurs, l'un des problèmes les plus importants de l'immeuble, à savoir des soucis récurrents d'humidité et de moisissures, ne semble pas avoir été traité.

Les différents éléments que l'on vient d'évoquer relèvent de préjudices de natures très différentes, tant par leurs origines (erreur technique, évolutions réglementaires, décisions de la municipalité non adossées directement au programme Magdolna), que par leurs conséquences (sur les finances, sur le confort). Du point de vue des acteurs, il n'en résulte pas moins un sentiment global de trahison, tant les promesses de la réhabilitation étaient nombreuses. Si nous nous en tenons aux deux mots d'ordres détaillés plus haut, à savoir « et » la lutte contre la taudification, « et » le maintien sur place des ménages les plus défavorisées, le compte n’y est pas, voire se présente déficitaire. L'accumulation des mauvaises surprises financières est particulièrement lourde pour des personnes qui se situent le plus souvent en bas de l'échelle salariale, contraintes de puiser dans le vivier du travail au noir pour boucler les fins de mois ${ }^{22}$. Elles appartiennent à un groupe social situé entre l'ancienne "classe moyenne kadarienne " (kádári kisemberek), dont elles partagent de nombreux traits culturels, et celle des «perdants de la transition » (leszakadtak, les « déclassés»), dans lequel la mobilité sociale est généralement descendante ${ }^{23}$. Plutôt protégées pendant la période communiste, ces personnes ont réussi à s'adapter de justesse à la précarisation de l'emploi au début des années 1990, sans pour autant parvenir à se constituer une situation confortable. Leur seul «patrimoine » est souvent le logement public qui leur a été attribué, dans la mesure où les règles régissant leur attribution garantissaient, dans la plupart des municipalités, un rapport au bien très proche de la propriété privée. De

22. Kolosi T., "Stratification and social structure in Hungary », Annual Review of Sociology, vol. 14, 1988, pp. 405-419.

23. Du nom de János Kádár, dirigeant de la République populaire de Hongrie entre 1956 et 1988, la classe moyenne kadarienne était le socle du régime socialiste, désignant un spectre social allant des ouvriers peu qualifiés des anciennes manufactures d'État aux petits fonctionnaires de l'administration centrale. Cette catégorie a été récemment exhumée par un rapport portant sur l'état de la société hongroise, dans lequel les auteurs insistent sur le déclin politique et socioéconomique de ses membres, cf. Péter R. et al.,Osztálylétszám 2014 [Effectifs de classe 2014], Budapest, GfKHungária; MTA Társadalomtudományi Kutatóközpont [Centre de recherche en Sciences sociales de l'Académie hongroise des Sciences], 2014, p. 201. 
fait, lorsque le programme de réhabilitation est lancé, beaucoup de logements rénovés sont déjà dans un bon état, car ayant été entretenus, souvent depuis plusieurs décennies, par les mêmes familles. Le sentiment de trahison est d'autant plus intense que les habitants ont souvent quitté des logements relativement confortables, qu'ils s'étaient entièrement appropriés. Au-delà de l'inévitable inconfort symbolique de la « refamiliarisation » avec l'espace domestique, laquelle repose sur une intériorisation cognitive, les malfaçons créent quant à elles un inconfort matériel entravant ${ }^{24}$. Au-delà de l'insécurité financière qui s'ouvre devant ces habitants, c'est aussi la « fonction restauratrice » du logement, particulièrement prégnante au sein des classes populaires, qui est ici touchée 25 .

\section{Surmonter les préjudices, demander réparation(s) Une mobilisation inscrite dans les pratiques ordinaires}

La mobilisation collective que nous allons décrire ici concerne un groupe de voisins au sein de l'immeuble enquêté, qui décident de porter l'objet du litige auprès de la municipalité. Les habitants mobilisés sont ceux qui habitaient déjà ici avant la réhabilitation. Ils se connaissent souvent depuis longtemps, ce qui montre bien ici la force des liens sociaux ancrés dans l'espace du proche, comme une ressource d'action rapidement mobilisable. Cette mobilisation s'inscrit avant tout dans un tissu de relations sociales "normales » forgé dans le temps long par les structures socio-spatiales ordinaires de la vie quotidienne ${ }^{26}$. Dans les grands immeubles à coursive budapestois, les discussions sur le pas de la porte du logement sont fréquentes et la porosité entre l'espace intime et extime permet facilement les visites entre voisins. Lorsque les premières factures de gaz arrivent, celles-ci deviennent ainsi très vite l'objet de toutes les conversations.

Le premier acte qui marque véritablement le début de la mobilisation est l'interpellation in situ des représentants de Rév8, dépêchés sur le terrain pour suivre l'avancement des ultimes chantiers de rénovation. Les témoignages des deux parties s'accordent pour souligner le caractère houleux des échanges, les uns comme les autres ayant l'impression d'être enferrés dans un « dialogue de sourds ». L'incompréhension réside dans le hiatus entre la visibilité institutionnelle de Rév8, considérée par beaucoup comme une « municipalité-bis »voire dans notre cas un bailleur social à part entière -, et son absence de

24. Sur la question de l'appropriation de l'espace et des dimensions idéelles et matérielles que cette notion implique, voir : Ripoll F., Veschambre V., «L'appropriation de l'espace : une problématique centrale... ", in Penser et faire la géographie sociale. Contributions à une épistémologie de la géographie sociale, Rennes, Presses universitaires de Rennes, 2006, pp. 295-304.

25. Schwartz O., Le monde privé des ouvriers, Paris, Presses universitaires de France, 1990.

26. Auyero J., «L'espace des luttes », Actes de la recherche en sciences sociales, ${ }^{\circ} 160,2005$, pp. 122-132. 
moyens pour régler les problèmes auxquels les habitants sont ici confrontés. Par ailleurs, les responsables qui font le déplacement sont souvent des professionnels de l'urbain (géographes, sociologues, économistes, architectes) peu préparés à ces situations conflictuelles.

«Sur tout ça, nous n’avons pas de réponse car chacun se renvoie la patate chaude... en disant que “pour ça, ça n'est pas à moi qu’il faut s'adresser mais à untel ou untel”, que “ce n'est pas moi le responsable”, que "c'est lui”, enfin tu vois, ce genre de choses... Personne n'est responsable de rien... 27 »

Face à l'absence de réponse satisfaisante, les riverains se tournent alors vers la municipalité, c'est-à-dire pris ensemble les élus et l'administration. Leur sollicitation exige un mode opératoire plus complexe, reposant sur la prise de rendez-vous au sein même du siège de la municipalité d'arrondissement situé à plusieurs centaines de mètres du quartier, laquelle prend selon les dires de plusieurs enquêtés les traits d'un véritable " parcours du combattant ».

«Quand nous nous rendons à la mairie [d'arrondissement] - j'ai des voisins qui ont été confrontés à ça - ceux qui osent demander pourquoi le chauffage est si élevé, que ce soit poliment ou non... eh bien les vigiles les mettent aussitôt dehors... 28 »

La prise de rendez-vous implique la négociation d'une rencontre durant les horaires d'ouverture des bâtiments officiels, impose des délais de réponse incertains, lesquels sont souvent difficilement conciliables avec une vie professionnelle ponctuée d'horaires alternés et le cumul de plusieurs petits emplois à temps partiel. La question de la ressource-temps comme clé de compréhension des inégalités entre habitants engagés dans un litige avec l'administration, se vérifie par le témoignage d'un riverain habitant un autre immeuble réhabilité du quartier. Celui-ci, par ailleurs tête de pont associatif à la forte personnalité, nous explique par le menu comment sa stratégie de harcèlement par le «pied de grue » devant les bureaux des élus et fonctionnaires municipaux avait porté ses fruits, notamment sur des questions de malfaçons similaires à celles rencontrés par les habitants enquêtés. Les inégalités s’accentuent également bien que marginalement - par des dispositions contrastées pour se repérer à la fois dans les méandres de l’administration municipale (qui solliciter, auprès de

27. Entretien avec une habitante réalisé en décembre 2013. Citation originale : «Nem kapunk rá választ. Mindig megy ez az egymásratologatás.. Hogymost ne engem kérdezzen, öt kérdezzék, hogy most nem én vagyok a felelös, ö a felel s, tehát érted ezek vannak. Nincsgazdája... ».

28. Entretien avec une habitante réalisé en décembre 2013. Citation originale : « Ha bemegyünk az önkormányzathoz, biztonságiörrel, vannak olyan lakótársaim. Aki megmeri kérdezni, hogy miért, miért ennyi a fütés, már kisértetik ki, amellett, hogy trágárul, vagy bármilyen módon megszólalna. ». 
qui demander un rendez-vous) et, au-delà, maîtriser le socle réglementaire minimal sur lequel appuyer ses demandes. Le militant associatif évoqué cidessus, se décrivant lui-même comme un déclassé issu de la petite bourgeoisie intellectuelle de province, parvient à ses fins par une connaissance fine des réglementations locales en vigueur et du jeu d'acteurs entre municipalité, bailleur locatif et régie municipale.

Pour ces habitants, il existe souvent une méfiance envers la langue juridico-administrative, laquelle aurait pour vocation principale de les induire en erreur. Au cours de nos entretiens, les riverains nous mettent souvent à profit pour comprendre les courriers qu'ils reçoivent de la part de la municipalité. Convaincus de leur «bon droit », leur sollicitation inquiète de notre regard révèle sans doute un objectif de certification de leur propre interprétation. Cette question de la certification d'un « bon droit » ou droit légitime par un tiers, indexée à la faible disposition à la compréhension du fonctionnement institutionnel, explique le recours à un avocat effectué par le groupe d'habitantes mentionnées plus haut. Ce recours ne doit pas être surinterprété comme une forme de judiciarisation des enjeux. Le rôle de l'avocat est ici de fournir une explication de texte et de rédiger les courriers de réclamation en bonne et due forme. La fonction symbolique que l'avocat endosse emprunte en partie au rôle dévolu aux écrivains publics, décrits par Franck Cochoy et Ygal Fijalkow comme des « assistances sociales bis » chargés de mobiliser « toute l'expérience accumulée dans la gestion des détresses sociales auprès des divers organismes pour imaginer des solutions et faire avancer les dossiers 29 ». Rencontré par l'intermédiaire d'une ancienne voisine victime d'une procédure d'exclusion, cet homme de loi est sensiblement rodé à affronter ces situations de blocage, mais sans pour autant les faire aboutir devant des juridictions compétentes.

On le voit ici, la mobilisation habitante est graduelle, au sens où elle nécessite le recours à des répertoires d'action de plus en plus spécifiques. Elle est également sinueuse, au sens où les riverains n'abandonnent pas nécessairement les techniques mobilisées au début du conflit. On retrouve là ce que Michel Offerlé analyse comme des logiques d'action à la fois plurielles et combinatoires 30 . Cela renvoie, d'une part, au «tâtonnement stratégique » de riverains souvent peu familiers du fonctionnement institutionnel et, d'autre part, à l'attitude de leurs interlocuteurs, lesquels semblent tous rejeter la responsabilité de la situation sur des tiers. Ce mouvement de défausse est particulièrement net entre la municipalité et la régie Rév8. Les premiers estiment que cette dernière est comptable dans la mesure où le litige porte sur les effets du pro-

29. Cochoy F. et Fijalkow Y., «Le marché de l'aide sociale : représentations, paradoxes et enjeux », Politiques et Management public, vol. 20, no 2, 2002, p. 117-141.

30. Offerlé M., "Retour critique sur les répertoires de l'action collective (XVIII - XXIe siècles) ", Politix, vol. 81, no 1, 2008, p. 181. 
gramme de réhabilitation, tandis que les seconds mettent en avant leur absence de moyen réglementaire pour agir. On touche ici un des principaux paradoxes de la réception sociale de l'urbanisme managérial, à savoir le contraste entre d'importants moyens pour «faire » et l'impuissance institutionnelle pour « gérer » l'après.

\section{L'épreuve de la visibilité forcée}

L'échec répété de ces stratégies par lesquelles la dimension collective n'est jusqu'à présent que secondaire, justifie le déplacement de la logique d'action des locataires mobilisés dans l'espace public. Ce déplacement emprunte les mêmes voies de mobilisation conventionnelle que le recours à la voie politicoadministrative tel que décrit plus haut : il se fait au sein des réunions publiques, organisées en marge du programme de réhabilitation par les associations de riverains ou par les pouvoirs publics. L'intervention dans ces arènes locales n'est pas ici liée à une volonté de «mise en lumière » ou de publicisation du litige, mais plutôt à une possibilité de faire se confronter les discours des différents opérateurs institutionnels mentionnés plus haut.

Ces réunions publiques sont héritées des dispositifs participatifs mis en place par le Programme Magdolna, lesquelles se sont trouvées appropriées par les associations de riverains créées au même moment, mais aussi pérennisées par les pouvoirs publics eux-mêmes. Quels que soient les organisateurs, ces réunions se déroulent souvent au même endroit - une ancienne fabrique de gants (keszttyügyár) transformée en maison de quartier - et suivent le même modus operandi. Face à l'assistance, s'assoient sur l'estrade les représentants de Rév8, de la municipalité d'arrondissement, parfois de la police nationale ou des leaders associatifs. Dans le public, on retrouve souvent des habitués qui, malgré leur effectif réduit, disposent souvent de puissants relais à l'intérieur du quartier. Prendre la parole dans une telle configuration scénique implique forcément une certaine intensité dramatique. Parmi le groupe de voisins, deux femmes ont décidé de faire entendre leur voix au nom de tous, parce qu'elles sont, selon leurs propres termes "plus communicatives que les autres ». Leur intervention n'obéit à aucun ordre du jour, ce qui lui confère une dimension subversive, rapidement atténuée par une attitude révérencieuse, qui trahit sans doute une volonté de ne pas faire de vagues, mais aussi un certain pragmatisme visant avant tout à régler ce qu'elles considèrent comme une situation de blocage. En présence d'un élu, le représentant de la régie municipale Rév8 est contraint de répondre en évitant de mettre en difficulté sa tutelle.

La mise en visibilité du litige n'a pas ici pour effet de clarifier le hiatus entre ces deux opérateurs, mais de créer un réflexe de protection mutuelle. Alors que les employés de Rév8 ont souvent, sur le terrain, un comportement empathique avec des habitants dont ils gèrent les délogements et relogements 
depuis le début du programme de réhabilitation, la réponse publique de leur représentant est une salve violemment ressentie. En s'appuyant sur un appareillage vidéo par lequel il montre le caractère spectaculaire des travaux de réhabilitation, il dénie le caractère légitime de la mobilisation des habitants en réduisant leurs incriminations à des demandes superfétatoires de personnes "qui en veulent toujours plus». Les éventuelles prises de parole des élus ou des représentants associatifs acquis à la cause de la municipalité démultiplient le plus souvent cet effet d'humiliation. Acculés, les habitants mobilisés persistent pourtant dans cette voie, mais en recourant cette fois à des personnalités identifiées comme qualifiées (dont la légitimité n’est pas discutée), pour « traduire » et « porter » le litige sur la place publique ${ }^{31}$. Ces personnalités étant souvent politisées, connues en tout cas comme des opposants à l'équipe municipale, cette stratégie du recours tend, comme nous le verrons dans la partie suivante, à déplacer la mobilisation habitante sur une ligne de crête entre simple « demande de réparation» et lutte «politique».

La visibilité forcée de la mobilisation produit dans un premier temps des effets délétères, notamment en renforçant le sentiment de trahison déjà latent au moment où les différents éléments composant le litige se sont révélés. Pour en prendre la mesure, il convient de rappeler que ces habitants ont été confrontés durant les années 1990 à un désengagement important des pouvoirs publics de la régulation de pans entiers de la vie sociale ${ }^{32}$. Jusqu'à la mise en place du programme Magdolna, l'attribution et la gestion des logements municipaux obéissaient à une logique qui oscillait entre paternalisme et clientélisme. L'irruption de la gestion managériale a sans doute permis de rompre avec certaines pratiques aux marges de la légalité, mais elle est rarement adaptée à la gestion de problèmes humains qui sortent des « dispositifs » conçus dans une logique de rationalité organisationnelle. Le sentiment de trahison sonne aussi comme un désaveu cinglant de la posture légitimiste et exemplaire adoptée par bon nombre de ces habitants dans leur rapport à la sphère politique. Il en résulte une « déformation de la reconnaissance 33 » qui ajoute à la demande de réparation, la lutte pour en reconnaître le caractère légitime.

31. Seguin L., "Faire entendre la parole des citoyens par le recours au film. Analyse d'un panel de citoyens dans la gestion de l'eau », Participations, vol.7, n³, 2013, pp. 127-149.

32. Concernant les logements municipaux, la situation est en tout point comparable à celle décrite par Bénédicte Florin dans les cités nassériennes (Florin B., "Des cités nassériennes aux villes nouvelles du désert : la fin du logement social ? ", in L'Égypte an présent, Paris, Actes Sud/Sindbad, 2011,p. 129-144). Dans ce chapitre, elle emploie également le terme de " trahison », emprunté à Anne Gotman pour illustrer le rapport particulier qui unit les locataires de logements sociaux et les pouvoirs publics (Gotman A., "L'accession à la propriété dans le parc social : quelle demande ?", Annales de la Recherche Urbaine, $n^{\circ} 65,1994$, pp. 66-67.).

33. Honneth A., op. cit. 


\section{Entre légitimation et stigmatisation, déjouer les déformations de la recon-} naissance

L'envolée des coûts de chauffage ne concerne que très peu d'immeubles réhabilités par le programme Magdolna. Alors que le problème des malfaçons est difficile à évaluer quantitativement, l'augmentation automatique des charges locatives concerne quant à elle tous les riverains occupant un immeuble réhabilité. Alors que l'on assiste, çà et là, à des expulsions de locataires pour impayés, l'opposition municipale ainsi que des associations pour le droit au logement participent à l'émergence d'un problème public sur le thème de la mise à la rue. Lorsque nous réalisons nos entretiens, la question de la politisation de la mobilisation se pose de façon aigüe. D’une certaine façon, la mise en visibilité du litige enjoint les riverains mobilisés à faire un choix sur la façon de poursuivre leur lutte : se trouver des alliés dans l'espace public ou continuer d'agir en explorant d'autres voies, plus discrètes, face aux pouvoirs publics.
«Avec les habitants, la situation c'est qu'une partie d'entre nous a pu revenir, dans la mesure où nous payions régulièrement nos loyers, mais nous n'avions jamais eu de problèmes les uns avec les autres, on essayait de faire bloc, etc. Mais malheureusement nous sommes en colère, parce que nous vivons sous pression, tout le monde a peur de devenir sans-abri, qui sera... Nous sommes déses- pérés au point que nous ne discutons plus avec plaisir de quoi que ce soit. De rien 34 ».

La question des alliances n'est pas anodine car elle renvoie à l'inscription de ces habitants dans un espace social fortement fragmenté sur le plan symbolique. Comme nous le rappelions dans la première partie, les habitants de l'immeuble enquêté appartiennent à un groupe social situé sur une ligne de crête entre l'ancienne classe moyenne kadarienne et les perdants de la transition. Si l'on s'en tient à des critères sociologiques objectivés - on retiendra surtout le profil socio-économique et la profession -, le groupe de voisins mobilisés est plutôt représentatif de l'ensemble des locataires municipaux du huitième arrondissement. Pourtant, lorsque nous les questionnons sur leur positionnement social, chacun d'entre eux insiste de la même façon pour se raccrocher culturellement aux classes moyennes tout en mettant à l'écart, parfois avec des discours très durs, les groupes les plus précarisés du parc locatif municipal. Cette façon de s'inscrire dans la topographie sociale est étroitement corrélée à un faisceau convergent de trajectoires biographiques, qui se distinguent à la fois par un capital scolaire et culturel plus important, mais aussi par la reven-

34. Entretien avec une habitante réalisé en décembre 2013. Citation originale : «A lakókkal hát az van, hogy mi egy páran így jöhettünk vissza, akik ilyen jól fizetö bérlök voltunk, nekünk soha nem is volt egymással probléma, igyekeztünk azért összetartani, meg satöbbi. De sajnos meg vagyunk keseredve, mert elnyomás alatt élünk, mindenki fél, hogy hajléktalan lesz, ki lesz... Es meg vagyunk keseredve, már nem szívesen beszélünk semmiröl. Semmiröl». 
dication d'une certaine citadinité. On retrouve là une forme très précaire de distinction sociale dont la fonction est ici de se préserver - par le comportement, la façon de vivre, le rapport aux autres - du risque de "glissement » (lecsuszás) dans la "pauvreté profonde " (mélyszegénység). Quant à ceux des locataires municipaux qui font l'objet de procédures d'expulsion, c'est souvent, selon ces riverains, "parce qu'ils l'ont mérité ».

Un autre élément de contextualisation, qui se confond parfois avec ce mécanisme de distinction, est celui de la peur du stigmate. En Hongrie, les rapports sociaux s'inscrivent dans un imaginaire souvent ethnicisé 35 . Au-delà même des appartenances ethniques, la question de la «pauvreté profonde » est souvent considérée comme un attribut de la «tsiganité ». Ce concept est délicat à manier dans la mesure où la «tsiganisation » (lecigányozás ${ }^{36}$ ) ne concerne pas tous les «dits Roms/Tsiganes » 37 et peut même être employée envers des «blancs». Dans les esprits, est «tsiganisé » celui qui se complairait dans une «culture de pauvreté 38 », qui n'arriverait pas à se sortir du putri (littéralement « gourbi »). Quelle que soit la couleur de leur peau, les riverains mobilisés ont tous conscience des représentations qui collent aux locataires des logements municipaux, ce qui leur vaut d'être régulièrement dénoncés durant des réunions de riverains comme les responsables de tous les maux dans le quartier ${ }^{39}$. Pour échapper à la «tsiganisation », entendue donc ici comme une forme culturellement déviante d'une situation sociale précaire, ces locataires tentent de conjurer le stigmate en revendiquant une certaine exemplarité dans l'attitude, la façon de se comporter, la manière de ne pas faire de bruit.

À l'aune de ce contexte de fragmentation de l'espace social local, on devine aisément les enjeux qui se posent en termes de reconnaissance. La commutation d'une mobilisation « ordinaire » en mobilisation « politique » supposerait l'acceptation même d'une condition sociale commune, élément indispensable

35. Fodor Zs, « Migránsok és kisebbségek ellen irányuló rasszizmus, etnikai diszkrimináció és kirekesztés a sportban : helyzetjelentés az Európai Unióból [Le racisme dirigé, la discrimination ethnique et l'exclusion envers les migrants et les minorités dans le sport : état des lieux dans l’Union européenne] ", Föld-rész, vol. 3, n³-4, 2010, pp. 103-105 ; Sik E., Simonovits B. (dir.), A diszkrimináció mérése [La mesure de la discrimination], Budapest, Université Loránd Eötvös, 2012.

36. Le suffixe le-désigne en hongrois une idée d'effectivité de l'action, mais peut aussi vouloir évoquer dans certains contextes, une idée de rabaissement, le signifiant aussi «vers le bas ».

37. Nous employons ici cette expression de façon à ne pas forcer les catégories. Nous faisons nôtre la prudence exprimée par les chercheurs du réseau Urba-Rom sur cette question. Voir : Legros O., Rossetto J., "La “question rom” en Europe aujourd'hui : Regards croisés et mises en perspectives ", Études tsiganes, ${ }^{\circ} 46,2013$, p. 4-25.

38. Sur cette question, dans un contexte rural : Ladányi J., Szelényi I., « La formation d'un sousprolétariat rom - Enquête historique sur la condition des Gitans dans un village d'Europe centrale », Actes de la recherche en sciences sociales, n¹60, 2005, pp. 66-87.

39. Lepeltier-Kutasi L., Transformations de l'ordre des légitimités locales et processus de différenciation socio-spatiale dans le quartier Magdolna à Budapest, Mémoire de master en géographie, EHESS, 2012. 
à la conscience d'intérêts communs. Or, ces riverains sont pris en étau entre une volonté de se tenir à l'écart des franges les plus basses du groupe de locataires municipaux, tout en subissant eux-mêmes le stigmate lié à leur statut d'occupant résidentiel. À la déformation institutionnelle de la reconnaissance exprimée plus haut s'ajoute un tarissement extrême des ressources d'action collective. Le refus de la politisation de leur lutte se traduit alors par un « retour dans l'invisibilité » et une progressive démobilisation collective.

Lorsque nous écrivons ces lignes, le litige n'est pas clos. À l'échelle de l'immeuble, la démobilisation collective renvoie chacun à ses propres capacités à gérer l'incertain. Pour une partie des locataires, cela passe par une posture de résistance formelle, qui se caractérise par une rhétorique de la menace (de grève des loyers notamment), sans que celle-ci soit à aucun moment mise à exécution. Cette forme de "résistance passive » se traduit néanmoins par des " arts de faire 40 » sous contraintes, lesquelles s'inscrivent dans le quotidien et renvoient à des tactiques, des ruses subtiles qui permettent des formes de réappropriations précaires de l'espace ${ }^{41}$. L'enjeu est de continuer de mener une vie quotidienne "normale », et ce en dépit de l'épée de Damoclès qui menace de tomber à tout moment. Pour d'autres, le repli sur la sphère individuelle exacerbe le constat d'une impossible échappatoire. Il s'agit pour ces derniers, plus que jamais, de réinventer la nécessité d'un ailleurs symbolique, qui compense les fonctions de « restauration » ou de « conservation de soi » que le logement ne peut plus garantir ${ }^{42}$.

D'une certaine façon, l'épreuve de la visibilité a pour effet de mettre en exergue un cloisonnement des possibles. Sur le plan symbolique cela se traduit par le piège des luttes pour la reconnaissance, lesquelles montrent bien les enjeux de la stigmatisation. La fragmentation extrême de l'espace social, le refus de la relation à l'autre, doivent être replacés, comme le suggère Norbert Elias, dans des contextes de rapports de pouvoirs où l'on retrouve les mécaniques élémentaires de domination et de fabrication de l'exclusion ${ }^{43}$. Dans la situation présente, la stratification au-delà de deux groupes, complexifie néanmoins la grille de lecture entre dominants et dominés, dans la mesure où ces riverains mobilisés sont, selon les situations, tantôt les uns, tantôt les autres. Ce qui est intéressant dans cette position intermédiaire, c'est ce paradoxe selon lequel ils ne jouissent ni pleinement des ressources des dominants, ni pleinement des ressources des dominés. On a finalement ici une situation qui se rapproche bien du sens originel du concept d'aliénation. Ce sentiment de dépossession de l'individu, cette perte de sa maîtrise biographique a également son corollaire spatial : c'est-à-dire une fragilisation extrême des moyens et des ressources de l'appropriation des lieux ${ }^{44}$.

40. De Certeau M., L'invention du quotidien, tome 1 : Arts de faire, Paris, Gallimard, 1990.

41. Ibid.

42. Deprez S., Vidal P., "L'habitat auto-construit aux limites de la ville : des classes populaires havraises en quête d'ailleurs ", Espaces et sociétés, vol. 156-157, n 1, 2014, pp. 85-108. 


\section{Conclusion}

Le programme de réhabilitation Magdolna a été conçu au début des années 2000 comme un programme exemplaire, censé compenser la prétendue violence nécessaire des transformations urbaines par une politique volontariste en matière de maintien des habitants sur place et d'inclusion aux processus de décision. Comme on l'a vu dans notre développement, l'idéologie managériale qui commandait cette conception de la médiation sociale, a vite atteint ses limites dès lors que les opérateurs institutionnels ont été amenés, non plus à mobiliser les acteurs pour faire advenir un projet, mais à gérer l'après. Ce qui aurait pu se limiter à des doléances vite réglées s'est peu à peu transformé en épreuve, dont les riverains impliqués ne sont pas sortis indemnes. La mise en visibilité forcée du conflit par une "gouvernance par dispositifs », a ainsi déplacé les enjeux du règlement du litige vers ceux de la reconnaissance d'une légitimité à agir. Alors que les habitants étaient pensés au cœur du projet de transformation du quartier, rien n'a vraiment été fait pour leur assurer les conditions matérielles d'une réalisation effective de ces promesses. Au contraire, au-delà des idéaux normatifs d'épanouissement et de réalisation de soi, la gestion de la mobilisation des riverains semble avoir généré davantage de souffrance et de vulnérabilité que d'émancipation.

On touche ici un angle mort des travaux sur les effets sociaux des politiques de renouvellement urbain, lesquels - notamment les tenants de la géographie radicale - partent souvent du principe que le maintien dans les lieux est à considérer de façon positive ${ }^{45}$. Axel Honneth rappelle pourtant avec justesse que les «menaces sur le social » résident le plus souvent hors de processus objectivables, notamment au sein des expériences morales de l'injustice. De ce point de vue, c'est davantage la façon dont les situations humaines sont considérées par le système institutionnel qui devrait primer dans l'analyse. Alors que de nombreux débats ont lieu dans la sphère académique hongroise pour penser les phénomènes socio-spatiaux en cours à Budapest, cet enseignement nous permet de formuler quelques hypothèses sur les enjeux à venir. Comme le souligne Zygmunt Bauman, la mondialisation se caractérise par une fragilisation des socialisations, des accords et des statuts sociaux. Cela produit une situation paradoxale par laquelle, dans les sociétés post-industrielles, ça n'est plus tant la «mobilité des pauvres » qui est synonyme de vulnérabilité,

43. Elias N., op. cit.

44. Ripoll F., Veschambre V., «L’appropriation de l'espace : une problématique centrale... », art. cit.

45. La littérature critique pointe avec justesse la façon dont la marginalisation spatiale des «indésirables » est étroitement liée à des politiques urbaines au service des classes moyennes (Cf. Clerval A. et Fleury A., " Politiques urbaines et gentrification, une analyse critique à partir du cas de Paris ", Espace politique, n8, 2009, mis en ligne le 15 novembre 2009, consulté le 10 mai 2016). Néanmoins, nous pensons que l'expérience profonde de l'injustice ne peut être réduite à la seule éviction institutionnelle, mais peut procéder de mécanismes plus fins de mise à l'écart. 
mais bien davantage leur fixité : «Certains peuvent quitter à volonté la localité, n'importe quelle localité. Les autres regardent désespérément la seule localité à laquelle ils sont attachés leur glisser des mains à grande vitesse 46 ». De ce point de vue, les politiques de réhabilitation, qui valent désormais comme l'alpha et l'oméga des stratégies de lutte contre la pauvreté, ne donnent pas les moyens adéquats, pour les habitants concernés, de sortir de leur captivité.

46. Bauman Z., Le cô̂t humain de la mondialisation, Paris, Hachette, 1999. 